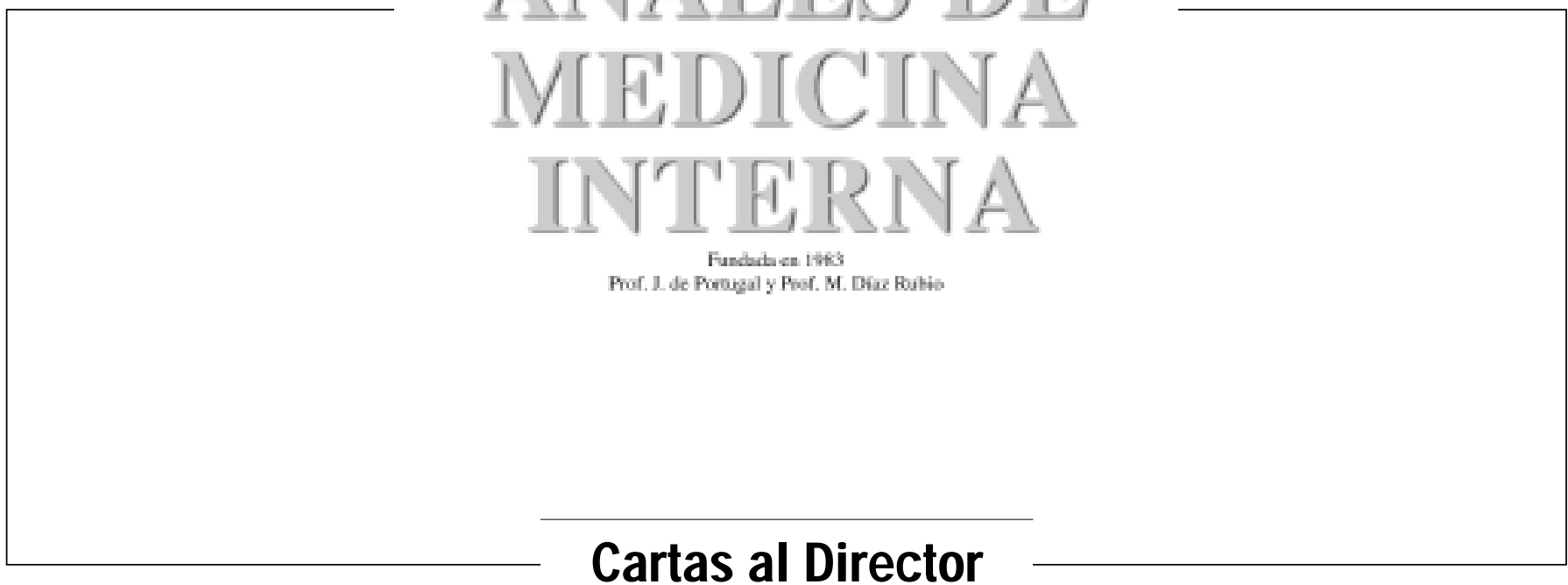

\section{Fasciolasis en el sur de Galicia. Presentación de dos casos}

\section{Sr. Director:}

La fasciolasis es una zoonosis de distribución universal con mayor incidencia en áreas rurales de bajo nivel higiénico-sanitario,donde el contacto entre hombres y animales infectados es continuo (1). EI agente etiológico es un trematodo,la fasciola hepática $(\mathrm{FH})$,que puede parasitar diferentes tipos de ganado herbívoro (ovino, vacuno ,caprino, etc) así como diversos animales (conejos, etc). EI hombre se contagia al consumir berros, u otro tipo de vegetales,infestados de metacercarias,que después de ingeridas,perforan el tubo digestivo para alcanzar el hígado y vías biliares donde maduran,y comienzan a eliminar huevos que pasan a las heces,completando el ciclo $(1,2)$.

Aunque ya ha sido descrita la infección por FH en nuestra comunidad autónoma (principalmente en el área de Lugo) $(3,4)$, pensamos que es de interés aportar dos nuevos casos de esta rara parasitosis.

Caso 1. Mujer de 48 años,ingresada para estudio de síndrome constitucional,cefalea holocraneal, y molestias abdominales, de 2 meses de evolución,con episodios aislados de escalofríos y fiebre en la última semana.Vive en zona rural con animales (gatos, perros, ovejas, etc.). Consumidora habitual de legumbres y hortalizas propias.En la exploración destaca hepatomegalia de $5 \mathrm{~cm}$ sin otros datos anormales.A su ingreso tenía:temperatura $39^{\circ} \mathrm{C}$, se extraen hemocultivos y se trata empíricamente con antibióticos.En el hemograma tenía,leucocitos $17.000 / \mathrm{mm}^{3}$, con $48 \%$ de eosinófilos (total $8.100 / \mathrm{mm}^{3}$ ), hemoglobina 10,8 gr \%, hematocrito $33 \%$, plaquetas $453.000 / \mathrm{mm}^{3}$ y VSG 118.En la bioquímica destacaba,fosfatasa alcalina $1.139 \mathrm{U} / \mathrm{L}$ y GGT $172 \mathrm{U} / \mathrm{L}$. Los hemocultivos extraídos a su ingreso fueron negativos. La radiografía de tórax, ecograffa abdominal y TAC cerebral fueron normales. Una gastroscopia demostró hernia de hiato. La búsqueda de parásitos en heces fue negativa, así como las serologías frente trichinella,equinococus granulosus y taenia solium. Una TAC abdominal evidenció hepatomegalia y múltiples lesiones hipotensas en lóbulo hepático derecho.La serología frente FH fue positiva a 1:2560. Se trató con praziquantel, a dosis habituales. Cinco meses después del tratamiento, la cifra de leucocitos era normal con $12 \%$ de eosinófilos. Una TAC abdominal 11 meses después del tratamiento no demostró anormalidad alguna.

Caso 2. Varón de 43 años, sin antecedentes de interés, consu- midor de berros procedentes de un mercado rural, en dos ocasiones en el último año. Es enviado para estudio de eosinofilia detectada en un hemograma. Refería como único dato destacable incremento de número de deposiciones (5/día) de características normales,en las últimas semanas. No tuvo fiebre, y no relataba dolor abdominal ni datos de síndrome constitucional. En el hemograma tenia, 23.480 leucocitos $/ \mathrm{mm}^{3}$, con $68 \%$ de eosinófilos (cifra total $15.900 / \mathrm{mm}^{3}$ ), VSG 80 y en la bioquímica destacaba, GOT $43 \mathrm{U} / \mathrm{L}$, GPT $51 \mathrm{U} / \mathrm{L}$, GGT $31 \mathrm{U} / \mathrm{L}$, fosfatasa alcalina $351 \mathrm{U} / \mathrm{L}$. EI resto de parámetros hematológicos, de coagulación y bioquímica eran normales. Los estudios de heces en busca parásitos fue negativa. La ecografía abdominal y la radiografía de tórax fueron normales. Se realizó una TAC abdominal que demostró la presencia de múltiples áreas hipodensas, de predominio en lóbulo hepático derecho (Fig. 1). Los anticuerpos anti-Fasciola hepática en suero por hemaglutinación pasiva fueron positivos a título 1:640. Recibió tratamiento con praziquantel a dosis habituales, constatándose meses mas tarde la desaparición de las lesiones hepáticas y normalización de cifra de eosinófilos.

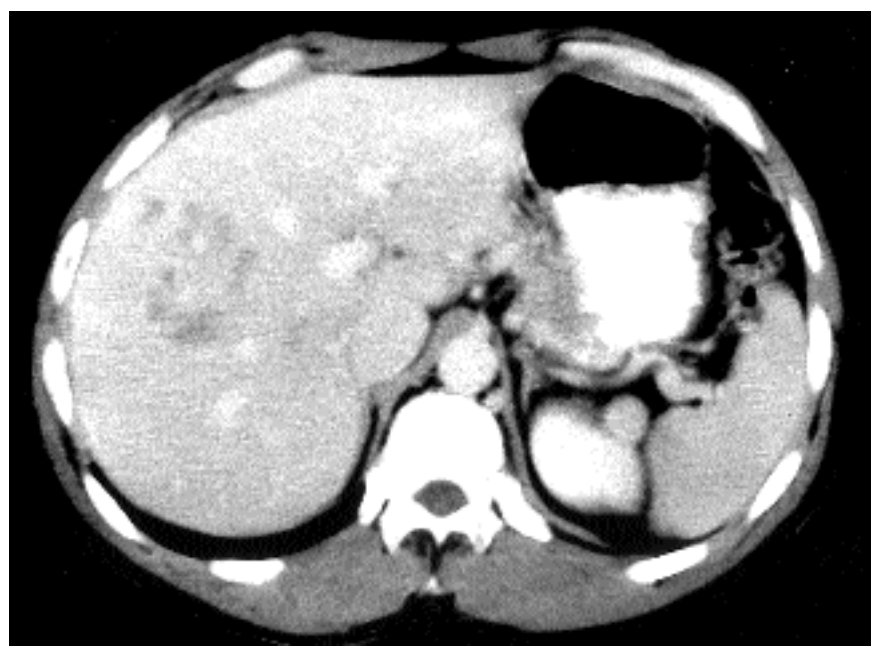

Fig. 1.

Desde las primeras descripciones de esta patología parasitaria en España, se han compilado en torno a 130 casos procedentes de forma mayoritaria de la mitad norte del estado, en relación direc- 
ta con la abundancia de ganado herbívoro fuente de contagio para el hombre (1-6).

Los dos nuevos casos aportados corresponden uno de ellos (caso 1) a la fase aguda de la enfermedad con presentación típica (fiebre, dolor abdominal,malestar general y adelgazamiento) con algún dato atípico (como es la cefalea persistente); mientras que el caso 2, representa la forma asintomática (2). En ambos casos existían antecedentes de ingestión de vegetales, y en uno de ellos se constató consumo de berros. Actualmente se piensa que cualquier vegetal, regado con aguas contaminadas por metacercarias, puede ser el origen de esta parasitosis para el hombre $(4,7)$.

La leucocitosis con eosinofilia marcada así como la elevación de la VSG y enzimas hepáticos indicadores de colestásis,presentes en ambos casos, se ajusta a las descripciones cínicas existentes de esta infección $(1,2)$. Así mismo coincidiendo con los datos de la literatura, la ecografía abdominal en los casos presentados fue normal, mientras que en la TAC, existían lesiones hipotensas sugestivas de fasciolasis hepática $(1,2)$.

Con la sospecha clínica basada en los datos clínicos, analíticos y radiológicos, la confirmación diagnóstica en nuestros casos se basó en test serológicos (hemaglutinación pasiva); en la fase aguda de la enfermedad, es infrecuente el hallazgo de huevos de parásitos en heces o aspirado duodenal, por lo que no se recomiendan para el diagnóstico $(1,2,7)$.

Aunque está descrita la ineficacia terapéutica del praziquantel en algunos casos de infecci6n por FH $(1,2,8)$, la respuesta clínica a dicha droga fue excelente en nuestros pacientes (desaparición de lesiones hepáticas,y tendencia a la normalización de los parámetros hematológicos alterados).

La sospecha diagnóstica de FH se debe establecer en pacientes con cuadro clínico compatible, procedentes del área norte del pais, $\mathrm{y}$ con historia de ingesti6n de berros u otro tipo de vegetales.

\section{J. Núñez Fernández, L. Anibarro García, L. Piñeiro Gómez-Durán}

\section{Servicio de Medicina Interna. Hospital Montecelo. Pontevedra}

1. Gargolas M, Torres R,Verdejo C et al. Infestación por Fasciola hepática. Biopatología y nuevos aspectos diagnósticos y terapéuticos. Enferm Infecc Microbiol Clin 1992; 10: 514-519.

2. Arjona R, Riancho JA, Aguado JM, Salesa R, González Macías J. Fasciolasis in developed countries: a review of classic and aberrant forms of the disease. Medicine (Baltimore) 1995; 74: 13-23.

3. López L, Alonso D, Iñiguez F, Mateos A, Bal M, Aguero J. Hepatic fasciolasis of long-term evolution: diagnosis by ERCP. Am J Gastroenterol 1993; 88: 2 118-2 119.

4. Pulpeiro JR, Armesto V, Varela J, Corredoira J. Fasciolasis:findings in 15 patients. Br J Radiol 1991; 64: 798-801.

5. Ayensa C, Muñoz J, Agud J, García F, Gaona T, Díaz R. Distomatosis por fasciola hepática. A propósito de 8 casos. Rev Clin Esp 1983; 168 : 261-265.

6. Campo JM, Milazzo A, Hebrero J, Sanz M, Revillo P, Lasierra J. Fasciola hepática. Presentación de 10 casos. Rev Clin Esp 1984; 173: 205-210.

7. Price TA, Tuazon CU, Simon GL. Fasciolasis: case report and review. Clin Infect Dis 1993; 17: 426-430.

8. Mora P, Gea F, Agud JL, Rabago L, Vargas E, de Juana P. Fasciolasis hepática. Resistencia al tratamiento con praziquantel y hallazgos radiológicos. Rev Esp Enferm Digest 1992; 82: 197-199.

\section{Agranulocitosis y ticlopidina}

\section{Sr. Director:}

Se presenta un caso de agranulocitosis en un paciente bajo tratamiento con ticlopidina, que motivó su ingreso en cuidados intensivos por sepsis. El paciente falleció a pesar de la recuperación hematológica.

Varón de 79 años con antecedentes de hipertensión, diabetes mellitus tipo II, limitación crónica al flujo aéreo, fibrilación auricular, hábito tabáquico y enolismo moderado. En 1991 presentó un ictus isquémico vertebro-basilar que se trató con ácido acetil salicílico (200 mg/día), obteniéndose la recuperación completa. En Septiembre de 1996 sufrió un nuevo episodio isquémico, por lo que se sustituyó el ácido acetil salicílico por ticlopidina (500 mg/día). Se recomendaron controles hematológicos cada 15 días durante los tres primeros meses de tratamiento. La aparición de neutropenia en el segundo control motivó la suspensión del fármaco.

Una semana más tarde el paciente comenzó con fiebre de $38^{\circ}$ y malestar general por lo que ingresó en el Servicio de Medicina Interna. En la exploración destacaban fiebre de $38^{\circ}$, palidez, leve deshidratación y ausencia de signos meníngeos. Auscultación cardiaca: arrítmica sin soplos; auscultación pulmonar: murmullo vesicular conservado. En el hemograma se objetivaron 1,12 x 10 $3 / \mu \mathrm{L}$ leucocitos (80 neutrófilos), $87 \mathrm{~g} / \mathrm{L}$ de hemoglobina, 27,2\% de hematocrito, $95,9 \mathrm{fL}$ de VCM y $128 \times 10^{3} / \mu \mathrm{L}$ plaquetas. Se obtuvieron cultivos de orina, esputo y sangre y después se instauró tratamiento antibiótico empírico con piperacilinal tazobactam (4 g/8 horas) y amikacina ( $500 \mathrm{mg} / 12$ horas). Se observó un empeoramiento de su estado general con insuficiencia respiratoria progresiva. Treinta y seis horas después del ingreso sufrió una parada cardio-respiratoria que obligó a intubación orotraqueal, masaje cardiaco y administración de adrenalina, recuperó ritmo cardiaco a los 5 minutos e ingresó en la unidad de cuidados intensivos en coma. El aspirado medular reveló un patrón de hiperplasia promielocitaria con más del $60 \%$ de dichos elementos celulares y subsiguiente paro madurativo. En los distintos cultivos realizados no se aisló ningún germen. En el control hematológico posterior se observó recuperación de la neutropenia (leucocitos: $4,52 \times 10^{3} / \mu \mathrm{L}$ con 1.898 de neutrófilos) sin mejoría clínica. Permaneció séptico, con insuficiencia renal anúrica, e inestable hemodinámicamente a pesar de dopamina a $20 / \mu \mathrm{g} / \mathrm{Kg} /$ minuto. El paciente falleció a los 5 días del ingreso hospitalario.

La ticlopidina es un antiagregante plaquetario que ha demostrado ser eficaz en la prevención del infarto cerebral en pacientes con episodios isquémicos cerebrales previos $(1,2)$.

La ticlopidina produce una elevada incidencia de reacciones hematológicas graves, como aplasia medular $(0,004 \%)$ y neutropenia $(2,3 \%)(3,4)$, de las cuales, el $0,8 \%$ son agranulocitosis $(5,6)$; también se han descrito trombopenias aisladas $y$, muy excepcionalmente, púrpura trombótica trombocitopénica.

El tratamiento con ticlopidina requiere control hematológico quincenal, durante los 3 primeros meses, para detectar estos efectos y disminuir su letalidad.

La actitud ante el desarrollo de neutropenia es la retirada de la medicación, y mantener un estrecho control clínico y analítico en un intento de prevenir un cuadro infeccioso $(7,8)$.

La prescripción de la ticlopidina debe ajustarse a las indicaciones autorizadas, ya que es un medicamento con elevada toxicidad hematológica (9).

\section{Fernández Esteban, B. Cuevas Ruiz*, Ma V. Cuevas Ruiz*, I. Saralegui Reta, J. Ochoa Gómez}

Servicios de Medicina Intensiva y *Hematología. Hospital. San Millán. Logroño

1. Flores Runk P, Raasch RH. Ticlopidine and antiplatelet therapy. Ann Pharmacoter 1993; 27: 1090-1098.

2. Shear NH, Appel C. Prevention of ischemic stroke. N Engl J Med 1995; 17: 460 . 
3. Farver DK, Hansen LA. Delayed neutropenia with ticlopidine. Ann Pharmacother 1994; 28: 1344-6.

4. Carlson JA, Maesner JE. Fatal neutropenia and thrombocytopenia associated with ticlopidine. Ann Pharmacoter 1994; 28: 1236-1238.

5. Legarra G, Beltrán de Heredia JM, Olabarna I, Menoyo MV, Villarreal JJ. Agranulocytosis caused by ticlopidine. Sangre 1994; 39: 229-30.

6. Ono K, Kurohara K, Yoshihara M, Shimamoto Y, Yamaguchi M. Agranulocytosis caused by ticlopidine and its mechanism. Am J Hematol 1991; 37: 239-42.

7. Geletko SM, Melbourne KM, Mikolich DJ. Pseudomonas bacteremia precipitated by ticlopidine-induced neutropenia. Ann Pharmacoter 1996; 30: $246-248$.

8. Johanning RJ, Tschida SJ. Pseudomonas bacteremia secondary ticlopidi ne-induced agranulocytosis. Ann Pharmacoter 1996; 30: 1201.

9. Lesesve JF, Callat MP, Lenormand B, et al. Hematological toxicity of ticlopidine. Am J Hematol 1994; 47: 149-50.

\section{Fiebre de Pontiac esporádica}

\section{Sr. Director:}

Las infecciones por bacterias del genero Legionella, denominadas en su conjunto legionelosis, se manifiestan básicamente de dos formas clínicas principales: como una neumonía, la denominada enfermedad de los legionarios, y como un síndrome febril sin focalidad de corta duración, sin afectación pulmonar y autolimitado, llamado fiebre de Pontiac. También está demostrada la infección asintomática y otras formas menos frecuentes secundarias a bacteriemia sin neumonía o la infección de una herida por exposición a agua contaminada (1). El motivo de esta carta es comunicar un caso de fiebre de Pontiac esporádico que, creemos de interés por su excepcionalidad.

Varón de 44 años que consultó por fiebre y cefalea. Entre los antecedentes personales destacaba diabetes mellitus, tuberculosis pulmonar 7 años antes y hepatitis aguda a los 18 años de edad. Tenía contacto con ganado vacuno y ovino. Tres días antes del ingreso comenzó con fiebre alta, cefalea global continua muy intensa, astenia y anorexia. La exploración física fue normal excepto por la presencia de fiebre. En la analítica destacaba ALT $160 \mathrm{U} / 1$, AST $168 \mathrm{U} / 1$, fostasa alcalina $337 \mathrm{U} / 1$, gamma-GT 86 U/1 y VSG 34. El hemograma, el análisis de orina, los hemocultivos, el urocultivo, rosa de Bengala, HbsAg, anticuerpos anti-virus de la hepatitis $\mathrm{C}$, la radiografía de tórax y de senos paranasales, la TAC encefálica y la ecografía abdominal fueron normales o negativos. Se le practicó punción lumbar; el líquido cefalorraquídeo, de aspecto macroscópico normal, contenía 290 × 10\%/1 hematíes (punción traumática), ausencia de leucocitos, proteínas $0,60 \mathrm{~g} / \mathrm{l}$ y glucosa $139 \mathrm{mg} / \mathrm{dl}$ (glucemia simultánea $268 \mathrm{mg} / \mathrm{dl}$ ). A su ingreso se instauró tratamiento sintomático con paracetamol y codeína, pese a lo cual persistió la fiebre y la intensa cefalea. El tercer día de ingreso, con la sospecha diagnóstica de fiebre Q, se inició tratamiento con doxiciclina, $100 \mathrm{mg}$ cada 12 horas, con excelente respuesta clínica, quedando apirético un día después. Tras un seguimiento de un año no hubo recidiva del cuadro. El estudio serológico de una pareja sueros, separados 3 semanas, fue negativo para C. burnetii, M. pneumoniae y C. psittaci. Se detectó seroconversión a Legionella pneumophila serogrupo 1 (negatividad en el suero de fase aguda y un título de $1 / 256$ en el de convalecencia).

La gran mayoría de casos de fiebre de Pontiac se han descrito en forma de brotes epidémicos. La presentación esporádica es rara; se desconoce si esto es una realidad u obedece a que es una enfermedad infradiagnosticada. En la literatura española sólo se han publicado dos casos $(2,3)$.
Se han descrito brotes de fiebre de Pontiac tras la exposición a torres de refrigeración, aire acondicionado, bañeras hidroneumáticas y fuentes decorativas (4-8) contaminados con bacterias del genero Legionella. La puerta de entrada es la vía respiratoria, a través de aerosoles contaminados. En nuestro caso no encontramos retrospectivamente la exposición a una posible fuente de infección.

La fiebre de Pontiac en una enfermedad febril aguda autolimitada, sin neumonía. En contraste con la enfermedad de los legionarios, la fiebre de Pontiac tiene un corto periodo de incubación (1-2 días contra 2-12 días), un alta tasa de ataque en las personas expuestas (>90\% contra el $1-7 \%$ ) y no se ha asociado con factores de riesgo. Los síntomas predominantes son fiebre, malestar general, mialgias y cefalea. Sólo requiere tratamiento sintomático y la recuperación es la regla en el plazo de una semana (1).

La sospecha diagnóstica debe surgir ante cuadros febriles autolimitados de presentación epidémica. En nuestro caso, el diagnóstico fue fortuito al practicar por error un estudio serológico para neumonía atípica cuando sólo se había solicitado serología para $C$. burnetii. El diagnóstico es siempre retrospectivo y por tanto su utilidad queda restringida para estudios epidemiológicos, careciendo de valor para el clínico que trata al paciente en la fase aguda. Se basa en la presencia de un cuadro clínico compatible y en la demostración de seroconversión o aumento de 4 veces del título de anticuerpos en el suero de convalecencia respecto al de fase aguda. Un título único igual o mayor de 1/256 se considera un diagnóstico de presunción de infección reciente. La existencia de varios serotipos de Legionella sin que exista reacción cruzada entre ellos y el hecho de que en la práctica asistencial, habitualmente, sólo se determinen los anticuerpos frente a Legionella pneumophila serogrupo 1, condiciona que algunos casos pasen desapercibidos (1). El microorganismo causal no se ha aislado nunca en los propios pacientes. Esto puede deberse a que dada la naturaleza benigna del cuadro es difícil justificar los procedimientos invasivos necesarios para obtener muestras apropiadas para cultivo en ausencia de tos productiva. También se ha sugerido que la fiebre de Pontiac podría estar causada por legionellas no viables (8) y que la patogenia dependería de hipersensibilidad a antígenos bacterianos más que a una verdadera infección. El conocimiento del agente responsable del brote epidémico surge del aislamiento del microorganismo a partir de la fuente de contagio. Se han descrito brotes causados por diversos serogrupos de L. pneumophila, L. micdadei, L. feelei y L. anisa $(1,6,7)$. La determinación del antígeno de Legionella en orina, podría ser útil para hacer un diagnóstico en la fase aguda de la enfermedad (8), aunque este sólo está disponible para el serogrupo 1 de L. pneumophila.

Se desconoce porqué la infección por microorganismos del genero Legionella produce en unos casos neumonía y en otros fiebre de Pontiac. Se han implicado diversos factores como el diferente tamaño del inoculo, diferentes modos de transmisión, el estado inmunitario del huésped (1) y la virulencia de la cepa (9). Se ha demostrado que en un mismo brote epidémico pueden presentarse simultáneamente ambas formas clínicas de la enfermedad (5).

\section{F. J. Espinosa Parra, A. Alemán Lorenzo, L. Pretel Serrano, S. Moreno Guillén*, F. Herrero Huerta}

Servicio de Medicina Interna y *Sección de Microbiología. Hos pital General Universitario J.M. Morales Meseguer. Murcia

1. Yu VL. Legionella pneumophila (Legionnaires disease). En: Mandell, Douglas and Bennett s principles and practice of infectious diseases. Ed. Mandell GL, Bennett JE, Dolin R. (4 edition). Churchill Livingstone 1995; 2087-2097.

2. Erice A, Romero-Vivas J, Rodríguez C, Buzón L, Bouza E. Fiebre de Pontiac. Med Clin (Barc) 1984; 82: 676-677. 
3. Antón E, García C, Martí J, Altuna E, Bustillo JM. Fiebre de Pontiac: presentación de un caso esporádico. Enf Infec Microbiol Clin 1989; 7 : 281

4. Tolentino A, Ahkee S, Ramírez J. Hot tube legionellosis. J Ky Med Assoc 1996; 94: 393-394.

5. Thomas DL, Mundy LM, Tucker PC. Hot tube legionellosis. Legionnaires disease and Pontiac fever after a point-source exposure to legionella pneumophila. Arch Intern Med 1993; 153: 2597-2599.

6. Fenstersheib MD, Miller M, Diggins C, et al. Outbreak of Pontiac fever due to Legionella anisa. Lancet 1990; 336: 35-37.

7. Fallon RJ, Rowbotham TJ. Microbiological investigations into an outbreak of Pontiac fever due Legionella micdadei associated with use of a whirlpool. J Clin Pathol 1990; 43: 479-483.

8. Friedman S, Spitalny K, Barbaree J, Faur Y, McKinney R. Pontiac fever outbreak associated with a cooling tower. Am J Public Health 1987; 77: 567-572.

9. Fields BS, Barbaree JM, Sanden GN, Morril WE. Virulence of Legionella anisa strain associated with Pontiac fever: an evaluation using protozoan, cell culture, and guinea pig models. Infect Immun 1990; 58: 3139 3142 .

\section{Diarrea como manifestación inicial de inmunocitoma intestinal productor de inmunoglobulina $\mathrm{M}$}

\section{Sr. Director:}

El inmunocitoma o linfoma linfoplasmocitoide es una neoplasia de estirpe linfoide cuya principal característica es la capacidad de elaborar inmunoglobulinas. En menos de un tercio de los casos la proteína sintetizada y liberada es Ig (inmunoglobulina) M monoclonal. Las formas de presentación más habituales son ganglionar, esplénica y oculocutánea (1-3). En cuanto al patrón histológico, se distinguen 3 variedades: linfoplasmocítico, linfoplasmocitoide y polimorfo; los dos primeros se incluyen en el grupo de los linfomas de bajo grado de malignidad, mientras que el polimorfo se encuadra en los de grado intermedio $(1,2)$. Presentamos un caso de inmunocitoma intestinal con varias peculiaridades: debut con localización intestinal, presentación de dos picos clonales de inmunoglobulinas, así como la presencia de alteraciones analíticas características.

Varón de 61 años con antecedente de enfermedad de Dupuytren. Presenta diarrea de 4 meses de evolución, con 4-6 deposiciones al día, sin productos patológicos, acompañada de astenia, anorexia y pérdida de peso. En la exploración física destaca desnutrición, escasas adenopatías axilares bilaterales de 1 centímetro de diamétro, no dolorosas y no adheridas a planos profundos, y palpación del bazo a $3 \mathrm{~cm}$. del reborde costal. En el hemograma el número de leucocitos es normal $\left(6,2 \times 10^{9} / \mathrm{L}\right)$, con hemoglobina de $121 \mathrm{~g} / \mathrm{L}$ y $164 \times 10^{9} / \mathrm{L}$ plaquetas. La velocidad de sedimentación globular en la primera hora es de $95 \mathrm{~mm}$. Los parámetros de coagulación también están alterados con una actividad de protrombina del $30 \%$ y un TTPA de 42 segundos (control 34 segs). En el proteinograma se observa una banda biclonal IgM, de 1,30 g/L en la cuantificación de inmunoglobulinas. Está constituída fundamentalmente por el tipo IgM-Lambda y, en pequeña cantidad, Ig G-Kappa. Hay proteinuria de Bence Jones IgMLambda. En el estudio inicial de la diarrea, el cultivo para bacterias y parásitos es negativo; el test de la D-Xilosa y las grasas en heces también son normales. Otras determinaciones analíticas anormales son las siguientes: ASLO 1209 UI; factor reumatoide $160 \mathrm{U}$ y hemaglutinación a Treponema pallidum positiva con un título de 1/5200. Las hormonas tiroideas están alteradas con una T4 total de 17,7 ng/dL; T4 libre de 3,92 ng/dL; T3 de 1,91
ng/mLy TSH de $0,42 \mu \mathrm{U} / \mathrm{mL}$. En la serie ósea radiológica cabe reseñar la presencia de lesiones osteolíticas en cráneo. La tomografia computarizada evidenció únicamente esplenomegalia. Continuando con el estudio de la diarrea, en el tránsito intestinal se ve imagen de engrosamiento de pliegues en yeyuno e íleon, con fragmentación del contenido. Después realizamos una biopsia intestinal, en la que aparecen unas vellosidades hipertróficas con ocupación de la lámina propia por histiocitos espumosos y masas de material eosinófilo homogéneo PAS positivo (Fig. 1 ); la tinción de Rojo Congo es negativa. La inmunohistoquímica muestra acúmulos de paraproteína que corresponden a cadenas ligeras Lambda. Una punción aspiración con aguja fina de uno de los ganglios axilares más representativos corresponde a tejido ganglionar normal. El medulograma es normal, con un $3 \%$ de células plasmáticas; sin embargo, en la biopsia de médula ósea se encuentra una infiltración nodular paratrabecular de una población heterogénea de elementos linfoides (inmunoblastos, células plasmáticas maduras con inclusiones hialinas intranucleares- y centrocitos). El paciente se trata con ciclos de Clorambucil/Prednisona, mejorando clínicamente.

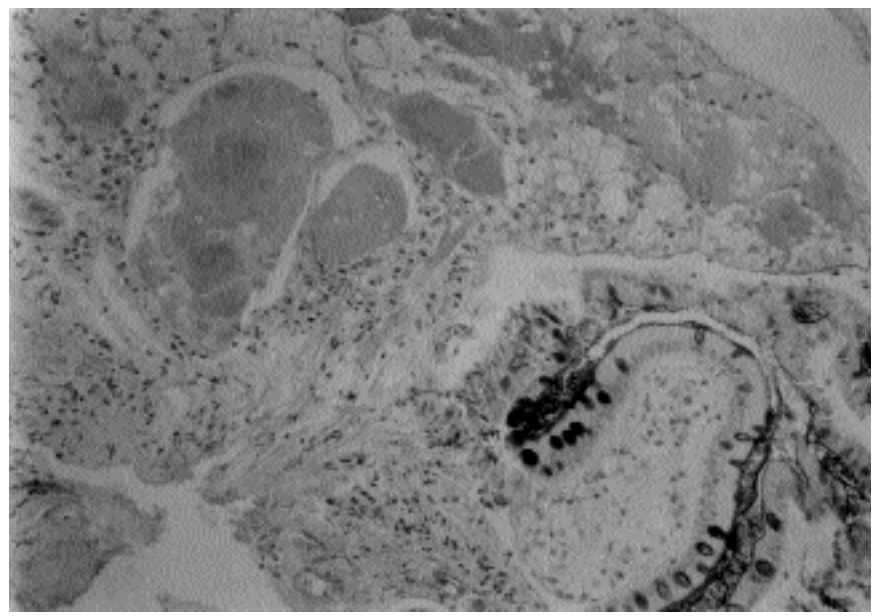

Fig. 1. Depósito de inmunoglobulina en mucosa duodenal. (P.A.S. $50 \mathrm{x})$.

En primer lugar, queremos acotar las denominaciones utilizadas en este artículo. El inmunocitoma o linfoma linfoplasmocitoide es un linfoma compuesto de linfocitos B y plasmocitos, capaz de elaborar inmunoglobulinas. Cuando la producción es de paraproteína IgM, puede etiquetarse, a la luz de la bibliografía, como Mieloma múltiple IgM o Macroglobulinemia de Waldenström. Estas denominaciones se aplican por las características peculiares dependientes de la producción de determinadas sustancias (tipo y cantidad de inmunoglobulinas fundamentalmente), de manifestaciones radiológicas (osteoporosis y lesiones líticas), del tipo de células y distribución de las mismas, del cuadro clínico y de la presencia de síndrome de hiperviscoidad. Con todo ello se construyen criterios diagnósticos para estas enfermedades de límite impreciso $(2,4,5)$. El caso que nos ocupa no reúne criterios para ser encuadradado como Mieloma múltiple IgM ni como Macroglobulinemia de Waldenström y por tanto nos quedamos con el diagnóstico más genérico de inmunocitoma. En segundo lugar, destacamos la baja afectación del intestino delgado en las discrasias de células plasmáticas. La extensión intestinal del mieloma es muy infrecuente, siendo poco común en la macroglobulinemia, aunque sí característica. En el caso presentado, la lesión intestinal era la primordial y además fue la diarrea la manifestación inicial de la enfermedad. El mecanismo de producción fundamental de la diarrea fue probablemente el depósito intestinal de paraproteína, hecho que se describe en pocas ocasiones en la bibliografía. Tam- 
bién llama la atención la poca expresión medular y en sangre periférica frente a la afectación intestinal (6-8). La existencia de un doble pico clonal de cadenas ligeras, Lambda y Kappa, no es infrecuente, suponiendo en algunas series hasta el $4 \%$ de las gammapatías clonales (9). Finalmente, queremos comentar las llamativas alteraciones analíticas que presentaba el paciente, con un factor reumatoide elevado, una hemaglutinación positiva frente a Treponema pallidum y alteraciones analíticas propias de hipertiroidismo, sin presentar clínica compatible con ninguna de estas entidades. Este hecho se explica por la "actividad anticuerpo" de la paraproteína, dando pruebas falsamente positivas mediante la forma de complejos con otras proteínas del plasma.

\section{J.A. Blázquez Cabrera, P. Lemberg*, F. Ortuño**, F. Mateos Rodríguez}

Servicio de Medicina Interna. *Servicio de Anatomía Patológica. Hospital de Hellín. Hellín (Albacete). **Servicio de Hematolo gía. Hospital General Universitario. Murcia

1. Woessner Casas A. Linfomas no Hodgkinianos. Rasgos generales y descripción de sus distintas variedades. En: Sans Sabrafen J. Ed. Hematología Clínica. Barcelona: Doyma SA 1982; 497-512.

2. Harris NL, Jaffe ES, Stein H et al. A Revised European-American Classiffication of Lymphoid Neoplasms: A Proposal From the International Lymphoma Study Group. Blood 1994; 84: 1361-1392

3. Gloor M, Seeling HP, Gehring W. IgM Kappa immunocytoma in Ig Mkappa monoclonal Ganmmapathy. Hautartz 1988; 39: 467-470.

4. Kyle R, Garton J. The spectrum of IgM monoclonal in 430 cases. Mayo Clin Proc 1987; 62: 699-731.

5. Patsouris E, Noel H, Lennert K. Lymphoplasmacytic/Lymphoplasmacytoid Immunocytoma with a High Content of Epithelioid Cells. Am J Surg Pathol 1990; 14: 660-670.

6. Cabrera A, de la Pva S, Pickren JW. Intestinal localizabon of Waldenstrom's disease. Arch Intem Med 1964; 114: 399-407.

7. Gad A, Willen R, Carlen B, Gyland F, Wickander M. Duodenal involvement in Waldenström macroglobulinemia. J Clin Gastroenterol 1995; 20: $174-176$

8. Tait RC, Oogarah PK, Houghton JB, Farrand SE, Haeney MR. Waldenström's macroglobulinemia secreting a paraprotein with lupus anticoagulant activity: posible association with gastrointestinal tract disease and malabsorption. J Clin Pathol 1993; 46: 678-680.

9. Pruzanski W, Underdown B, Silver EH, Katz A. MacroglobulinemiaMyeloma Double Gammopathy. Am J M 1974; 57: 259-266.

10. Merlini G, Farhangi M, Osserman EF. Monoclonal immunogbbulins with antibody activity in myeloma, macroglobulinemia and related plasma cells dyscrasias. Semin Oncol 1986; 13: 350365.

\section{Mastocitosis sistémica y resonancia magnética}

\section{Sr. Director:}

Galán y cols., presentaron en la revista que Ud. dirige un caso de mastocitosis sistémica (MS) con hepatomegalia y alteraciones digestivas, seguida en el tiempo de una leucemia mieloide secundaria (1). Los autores destacan la heterogenicidad e inespecificidad de la clínica acompañante, la similitud con los síndromes mieloproliferativos y la escasez de medidas terapéuticas efectivas, lo cual resalta importancia de la sospecha clínica, aspecto que es resaltado igualmente por otros autores (2-5). Al respecto nos gustaría ampliar la discusión del caso aportando nuestra experiencia terapéutica y diagnóstica con la utilidad de la resonancia magnética $(\mathrm{RM})$ en esta patología.
En primer lugar, desde la primera descripción en 1992 de la utilidad del tratamiento con alfa-interferón (IFN) en la MS, se han producido varias descripciones de casos aislados o pequeñas series, hasta que en un trabajo recientemente publicado (7) se valora el tratamiento con alfa-interferón (IFN) en 6 pacientes afectos de MS durante un año, objetivándose reducción del grado de infiltración de la médula ósea (MO), mejoría de la sintomatología infiltrativa y control de los cuadros mediados por citoquinas. Si bien, como indica Galán (1), no existe un tratamiento curativo, y es todavía pronto para afirmar su eficacia terapéutica, debido al buen control de la clínica acompañante, la objetivación de remisiones completas e incluso corrección de las alteraciones radiológicas, nuestro grupo de trabajo (7), como otros nacionales (8), consideramos al IFN como tratamiento de elección en las formas agresivas de MS.

Por otro lado, desde el punto de vista radiológico, en otra reciente revisión (9), se constataba alteraciones óseas en el 86\% de los pacientes. La mayoría presentaban osteopenia, aunque sólo se consideró patológica en relación con la edad en el 35,7\%; seguida de la presencia de esclerosis en el $43 \%$, de alteraciones osteoblásticas en el 29\%, y de lesiones líticas en el 14\%. La coexistencia de diferentes tipos de lesión se relacionó con cuadros agresivos de MS (9).

Con relación a la utilidad de la RM el diagnóstico de extensión, pronóstico y monitorización de respuesta terapéutica, se han comunicado en los últimos años varios patrones (10-13), dónde se ha intentado correlacionar el grado de infiltración, tipo de enfermedad, manifestaciones clínicas y pronóstico. Avila y cols. (10) estudiando únicamente pelvis con un equipo de 0,5T Picker con SE T1 y T2* describen la alta sensibilidad y especifidad en el diagnóstico de la infiltración de la MO, pero no logran correlacionar los hallazgos con los parámetros buscados. Nuestro grupo de trabajo, desarrolló un protocolo de estudio (11): coronal SE T1 (TR $60 \mathrm{~ms}$, TE 20msg) pelvis, columna vertebral, cabezas femorales y humerales en una unidad de RM 0,5 T (MAX 0,5 T General Electric), describiendo una clasificación del patrón de infiltración (Tabla I) con las denominaciones de Homogéneo (Fig. 1A) y No Homogéneo (subtipos difuso, moteado y reticulado) (Fig. 1B). Con este protocolo se estableció un "score" sumando los valores obtenidos de las diferentes áreas estudiadas, y dónde se obtienen unos valores de especifidad y sensibilidad similares a los descritos por Avila (10), y se estudia la correlación entre el patrón de infiltración medular en RM y el \% de mastocitos en la biopsia de MO, así como la correlación entre el grado de infiltración medular en RM y el estadio o categoría clínica de la enfermedad. En el primer supuesto la correlación fue baja; sin embargo, sí se observó entre el grado de infiltración medular en RM y la forma clínica (13).

TABLA I

PATRO NES DE INFILTRACIÓN M EDULAR EN RM Y "SCORE" CO RRESPO NDIENTE

\begin{tabular}{lll}
\hline Patrón & Subtipo & Score \\
\hline Homogeneo & & 4 \\
No homogéneo & & \\
& Difuso & 3 \\
& Moteado & 2 \\
Normalidad & Reticular & 1 \\
\hline
\end{tabular}

En conclusión, sin duda consideramos que el diagnóstico de la MS resulta muchas veces difícil de obtener debido a la amplia 


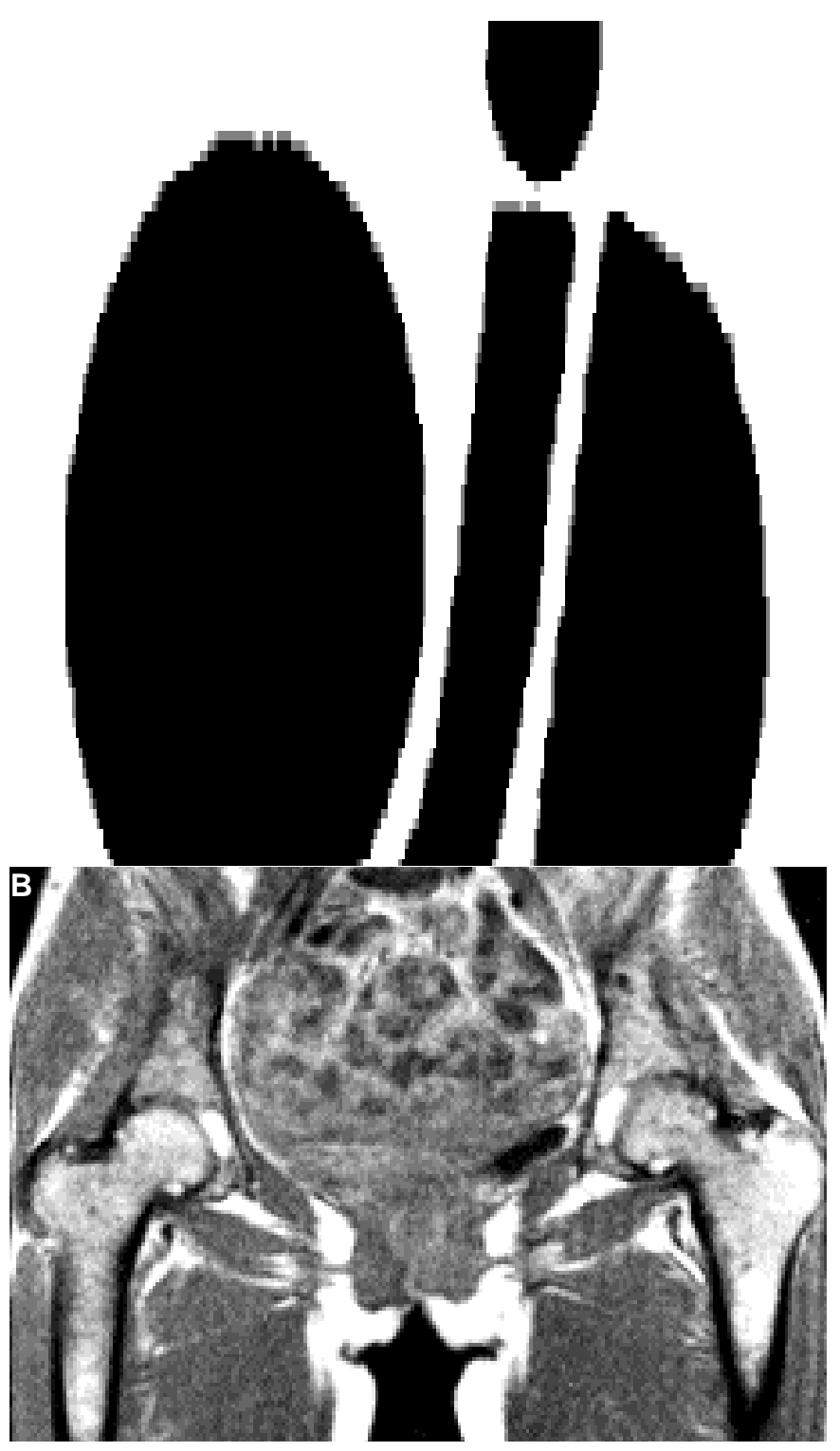

Fig. 1A. Columna SE T1 Sagital. Infiltración homogénea de médula ósea.

Fig. 1B. Pelvis SE T1 Coronal. Infiltración No Homogénea M oteada de huesos pélvicos y fémures.

variedad de manifestaciones clínicas y al curso indolente de la mayoría de los pacientes (1-6). La valoración multidisciplinar individualizada de cada paciente por parte de los dermatólogos, radiólogos, hematólogos, patólogos e internistas es indispensable para reconocer adecuadamente las diferentes formas clínicas de la enfermedad (8). La RM es una herramienta que cada día se revela de mayor utilidad en la evaluación de la infiltración de la MO en el estudio de extensión de procesos hematológicos (8-11), así como para obtener una muestra para diagnostico dirigido y en la valoración de la respuesta al tratamiento. Por tanto, su inclusión sistemática en el estudio de extensión de la MS está hoy día refrendada por la experiencia previa $(8,10-13)$. Por el momento, no disponemos de un tratamiento curativo de la MS, sin embargo, cuando los tratamientos sintomáticos no son eficaces, la opción de administrar IFN sería en ocasiones beneficiosa en el control de los síntomas y reversión de la infiltración $(1,2,6,7,8)$.

\section{J. A. García Erce, M. Roca Espiau*, P. Giraldo Castellano}

Servicios de Hematología y *Radiología. Hospital Universitario Miguel Servet. Zaragoza

1. Galán Alvarez P, Ibáñez García J, Funes Vera C, et al. Mastocitosis sistémica y leucemia mieloide aguda secundaria. An Med Interna (Madrid) 1999; 16 (9): 466-8.

2. Bernhard JD. Mastocytosis: for better and worse. Lancet 1996; 347: 705

3. Ribera JM, Feliú E. Mastocitosis sistémicas. Sangre (Barc) 1991; 36: 299-306.

4. Giralt M, Buñuel C, Rubio D et al. Mastocitosis sistémica: estudio de cinco casos. Sangre (Barc) 1987; 32: 299-310.

5. García Erce JA, Giraldo P, Vicente P, et al. Mastocitosis sistémica: estudio de 14 casos. Med Clin (Barc) 1998; 111 (13): 499-502.

6. Kluin-Nelemans HC, Jansen JH, Breukeleman H, Wollthers BG, Kluin $\mathrm{PM}$, Willemze R. Response to interferon alfa-2b in a patient with systemic mastocytosis. N Engl J Med 1992: 336: 619-623.

7. Giraldo P, García-Erce JA, Alvarez R, et al. Alfa-interferón y mastocitosis sistémica. Análisis de la eficacia terapéutica en seis casos. Rev Clin Esp 1998; 198: 345-350.

8. Escribano L, Bravo P, Bellas C, et al. El mastocito y las mastocitosis. Haematologica 1999; 84: 47-53

9. García-Erce JA, Giraldo Castellano MP, Roca Espaiu M. Alteraciones radiológicas en la mastocitosis sistémica. Radiología 1998; 40: 424-425.

10. Avila N, Ling A, Metcalfe D, et al. Mastocytosis: magnetic resonance imaging patterns of marrow disease. Skeletal Radiol 1998; 27: 119-126.

11. Mota J, Roca M, Giraldo, Erce JAG, Pereda RG. Systemic mastocytosis: MR imaginig of bone marrow involvement. Radiology 1997; 205: 632.

12. Giralt M, Roca M, Giraldo P, García-Erce JA, Alvarez R, Mayayo P, Rubio-Félix D. Evaluation of bone marrow involvement by magnetic resonance imaging in systemic mastocytosis. Blood 1997; 90: 2880.

13. Roca M, Mota J, Giraldo P, García-Erce JA. Systemic mastocytosis: MRI of bone marrow involvement. Eur Radiol 1999; 9 (6): 1094-7.

Etiopatogenia de los síndromes hereditarios de fiebre periódica

\section{Sr. Director:}

En un artículo reciente Medrano y Bruscas realizan una magnífica revisión del síndrome de hiperinmunoglobulinemia $\mathrm{D}$ y fiebre periódica (SHID) (1). Éste es un cuadro poco frecuente, pero que probablemente en ocasiones no se llega a diagnosticar por ser aún poco conocido. En el trabajo citado se hace énfasis en su diagnóstico diferencial con otros síndromes de fiebre recurrente y, en especial, con la fiebre mediterránea familiar (FMF), con la que comparte un patrón hereditario autosómico recesivo. Sin embargo, se afirma que la etiopatogenia del SHID es desconocida, lo que se explica por la fecha de aceptación del artículo.

Actualmente se incluye al SHID, junto con la FMF y la llamada fiebre hibernesa, en un grupo nuevo de enfermedades denominado como síndromes hereditarios de fiebre periódica $(2,3)$. Todos ellos se caracterizan por su carácter hereditario, que es, como se ha dicho, autosómico recesivo en la FMF y el SHID y autosómico dominante en la fiebre hibernesa. Además, comparten la aparición de fiebre recurrente, aunque con un inicio, periodicidad y duración variables, así como la producción de síntomas inflamatorios localizados en distintos órganos, cuyo patrón también es característico (Tabla I).

La base genética de la FMF quedó claramente dilucidada en 1997 con la clonación simultánea por un grupo francés y otro americano de un gen localizado en el brazo corto del cromosoma 16 y que se denominó MEFV (Mediterranean Fever) $(4,5)$. Este gen codifica una proteína llamada marenostrina (de mare nostrum, por el grupo francés) o pirina (de pyros, fiebre, por el americano), que actúa como un factor de transcripción génica 
CARACTERÍSTICAS Y ETIO PATO GENIA DE LOS SÍNDRO M ES HEREDITARIOS DE FIEBRE PERIÓ DICA

\begin{tabular}{llll}
\hline & $\begin{array}{l}\text { Fiebre M editerránea } \\
\text { Familiar }\end{array}$ & $\begin{array}{l}\text { Síndrome de HiperlgD } \\
\text { y Fiebre Periódica }\end{array}$ & Fiebre hibernesa \\
\hline No Familias afectadas & 10.000 & 100 & 10 \\
Herencia & $\begin{array}{c}\text { Autosómica } \\
\text { recesiva }\end{array}$ & $\begin{array}{c}\text { Autosómica } \\
\text { recesiva }\end{array}$ & $\begin{array}{c}\text { Autosómica } \\
\text { dominante }\end{array}$ \\
Edad de Inicio & $<20$ años & $\begin{array}{l}\text { Infancia } \\
\text { Variable }\end{array}$ \\
Duración de Episodios & $1-4$ días & $3-7$ días & $>1$ semana \\
Linfadenopatía & - & ++++ & ++ \\
Dolor Abdominal & ++++ & +++ & ++ \\
Dolor Torácico & ++++ & - & ++ \\
Artralgias-Artritis & +++ & +++ & $-/+$ \\
M ialgias & - & - & +++ \\
Rash Cutáneo & $-/+$ & +++ & +++ \\
Conjuntivitis & - & - & +++ \\
Amiloidosis & +++ & - & $-/+$ \\
Gen con mutaciones & M EFV & M VK & TNFR1 \\
Cromosoma afectado & 16 & 12 & 12 \\
Proténa codificada & Marenostrina/ pirina & M evalonato cinasa & Receptor de TNF \\
Tratamiento & Colchicina & Ninguno & ¿Esteroides? \\
\hline El número de cruces refleja la frecuencia relativa del trastorno & &
\end{tabular}

El número de cruces refleja la frecuencia relativa del trastorno.

específico de los polimorfonucleares, regulando su respuesta a la inflamación. Determinadas mutaciones en el gen favorecerían los mecanismos inflamatorios y la fiebre en los pacientes con FMF.

En 1999 se han publicado los estudios genéticos que caracterizan los trastornos moleculares y la etiopatogenia de los otros dos síndromes. En el caso de SHID se ha comprobado que los pacientes presentan mutaciones en el gen del cromosoma 12 que codifica la enzima mevalonato cinasa, cuya actividad es deficitaria y se excretan cantidades anormalmente elevadas de ácido mevalónico en orina durante las crisis de fiebre $(6,7)$. Precisamente, algunos compuestos, como los fosfatos isoprenilados, derivados de la ruta metabólica del ácido mevalónico han demostrado participar en mecanismos de transmisión de señal celular, que intervienen también en las respuestas inflamatorias de los neutrófilos (8). De esta manera, la elevación de la inmunoglobulina D sería únicamente un epifenómeno con utilidad como marcador diagnóstico del síndrome.

Finalmente, la fiebre hibernesa o fiebre recurrente autosómica dominante, que se ha descrito en un pequeño número de familias, la mayoría de origen irlandés (9), se demostró ligada a otro gen también localizado en el cromosoma 12. Este gen codifica el receptor tipo 1 del factor de necrosis tumoral (TNFR1). Los pacientes con mutaciones en este gen presentan mayor expresión de estos receptores en las membranas de los leucocitos, a la vez que una menor concentración de su forma soluble o libre en el plasma, cuya función parece ser antagonista de una excesiva respuesta inflamatoria (10).

De todo lo expuesto, se puede concluir que este grupo de enfermedades representa un importante reto diagnóstico para el clínico, al que sólo se puede enfrentar con un índice alto de sospecha y familiarizándose con sus formas de presentación. Sin embargo, a la vez, los conocimientos adquiridos sobre su etiopatogenia proporcionan excelentes modelos naturales, mediante los cuales se puede profundizar en las bases moleculares de la respuesta inflamatoria y facilitar nuevas dianas terapéuticas para el desarrollo farmacológico.

\section{J. de Dios García-Díaz}

Servicio de Medicina Interna. Hospital Universitario Príncipe de Asturias. Universidad de Alcalá. Alcalá de Henares. Madrid

1. Medrano San Idelfonso M, Bruscas Izu C. Síndrome de hipergammaglobulinemia D. An Med Interna (Madrid) 2000; 17: 213-6.

2. Centola M, Aksentijevich I, Kastner DL. The hereditary periodic fever syndromes: molecular analysis of a new family of inflammatory diseases. Hum Mol Genet 1998; 7: 1581-8.

3. Grateau G, Drenth JPH, Delpech M. Hereditary fevers. Curr Opin Rheumatol 1999; 11: 75-8

4. The French FMF Consortium. A candidate gene for familial Mediterranean fever. Nat Genet 1997; 17: 25-31.

5. The International FMF Consortium. Ancient missense mutations in a new member of the RoRet gene family are likely to cause familial Mediterranean fever. Cell 1997; 90: 797-807.

6. Houten SM, Kuis W, Duran M, De Koning TJ, Van Royen-Kerkhof A, Romeijn GJ, et al. Mutations in MVK, encoding mevalonate kinase, cause hyperimmunoglobulinaemia D and periodic fever syndrome. Nat Genet 1999; 22: 175-7.

7. Drenth JPH, Cuisset L, Grateau G, Vasseur C, Van der Velde-Visser SD De Jong JGN, et al. Mutations in the gene encoding mevalonate kinase cause hyper-IgD and periodic fever syndrome. Nat Genet 1999; 22: 17881.

8. Levy BD, Petasis NA, Serhan CN. Polyisoprenyl phosphates in intracellular signalling. Nature 1997; 389: 985-90.

9. McDermott EM, Smillie DM, Powell RJ. Clinical spectrum of familia Hibernian fever: A 14-year follow-up study of the index case and extended family. Mayo Clin Proc 1997; 72: 806-17.

10. McDermott MF, Aksentijevich I, Galon J, McDermott EM, Ogunkolade $\mathrm{BW}$, Centola M, et al. Germline mutations in the extracellular domains of the $55 \mathrm{kDa}$ TNF receptor, TNFR1, define a family of dominantly inherited autoinflammatory syndromes. Cell 1999; 97: 133-44. 


\section{Síndrome hipergammaglobulinemia D}

\section{Sr. Director:}

Hemos leído con atención la interesante revisión recientemente publicada en su Revista sobre el síndrome hipergammaglobulinemia D (1). Como los Drs. Medrano San Ildefonso y Bruscas Izu señalan, en el diagnóstico diferencial es necesario tener en cuenta que existen diferentes procesos que pueden cursar con aumento de la Ig D. Entre dichos procesos creemos que es importante citar a la enfermedad de Behçet. Por una parte, el síndrome hipergammaglobulinemia D puede, en ocasiones, presentar manifestaciones clínicas semejantes e imitar a la enfermedad de Behçet. Por otra, los niveles de la Ig D están elevados en un pequeño porcentaje de pacientes con enfermedad de Behçet en actividad. De hecho, ya que en la enfermedad de Behçet no existen signos o síntomas ni datos de laboratorio patognomónicos, algunos autores (2) han sugerido que la determinación de la Ig D, y su valor elevado, podría servir de ayuda en el diagnóstico de los casos complicados.

\section{F. J. Fernández Fernández, F. S. Martínez Debén, P. Sesma*}

Servicio de Medicina Interna. Hospital Arquitecto Marcide. Ferrol. La Coruña. *Departamento de Medicina. Universidad de Santiago de Compostela

1. Medrano San Ildefonso M, Bruscas Izu C. Síndrome hipergammaglobulinemia D. An Med Interna (Madrid) 2000;17: 213-6.

2. Sakane T, Takeno M, Suzuki N, Inaba G. Behçet's disease. N Engl J Med 1999; 341: 1284-91.

\section{Consentimiento y confidencialidad en la práctica médica del SIDA}

\section{Sr. Director:}

He leído con interés el artículo publicado en su revista (1) titulado Aspectos éticos en la práctica médica en el SIDA, cuyos planteamientos me parecen acertados en general, aunque creo que algunas consideraciones referentes al secreto profesional pueden generar confusión y malentenderse como recomendaciones.

Los autores parecen dudar que las pruebas de detección de anticuerpos frente al VIH precisen siempre de consentimiento informado. Un repaso de la ley no permite duda. El test para el VIH es una técnica médica diagnóstica, que determina en caso de positividad una de las informaciones más trascendentes por sus consecuencias médicas, familiares y socio-laborales, que un ser humano puede recibir de un médico. Por ser una información tan sensible para el paciente no cabe duda que está sometida al consentimiento informado, preferiblemente por escrito, en aplicación de la Ley General de Sanidad (2) artículo 10,6. Desde la ética, el respeto por la intimidad de las personas y la discriminación social que causa su positividad, determina que exista un acuerdo unánime en que, como norma debe informarse al paciente y pedirle consentimiento. Ese respeto debe ser real, y en caso de denegar su realización, derecho y ética obligan a respetar su deseo. Aunque sabemos que toda norma, puede tener excepciones, que no invalidan la norma. La propia Ley General de Sanidad (2) detalla las excepciones: cuando no está capacitado para tomar decisiones (en cuyo caso le corresponde a sus familiares o personas allegadas), cuando la urgencia no permita demora por poderse ocasio- nar lesiones irreversibles o existir peligro de fallecimiento (situación excepcional en la realidad) o cuando la no intervención suponga un riesgo para la salud pública (argumento no válido en casos individuales).

Todos estamos de acuerdo en que es deseable que para evitar su contagio sea el paciente quien notifique su enfermedad a su pareja, evitando al médico un grave dilema ético, elegir entre respetar el secreto profesional o informar del riesgo a su pareja. En caso de romper el médico el secreto profesional, la norma legal es clara. La Ley Orgánica 1/1982 (3) de 5 de mayo, articulo 7 dice, "se considera intromisión ilegítima, en el ámbito de la protección de la intimidad, la revelación de datos probados de una persona o familia, conocidos a través de la actividad profesional y oficial de quienes los revelan". Y el nuevo Código Penal (4) en su articulo 199 dice." 1. El que revelare secretos ajenos de los que ten ga conocimiento por razón de su oficio o sus relaciones labora les, será castigado con la pena de prisión de 1 a 3 años y multa de 6 a 12 meses. 2. El profesional que incumpliendo su obliga ción de sigilo o reserva, divulgue los secretos de otra persona será castigado con la pena de prisión de uno a cuatro años, mul ta de 12 a 24 meses e inhabilitación para dicha profesión por tiempo de 2 a 6 años". Por otra parte, en el caso hipotético de presentarse denuncia por haber contraído el VIH durante una relación sexual voluntaria entre adultos, el nuevo código penal lo consideraría posiblemente un simple delito de lesiones (5). Contrasta la dureza y desproporción con que se castiga al que se arriesga con la ruptura del secreto profesional, frente a la que recibiría el contagiador. De lo que no debe quedarnos duda es que romper el secreto profesional nos podría llevar a una situación legal complicada, que no es fruto de leyes antiguas, sino recientemente revisadas.

Desde la ética es más complejo, por un lado nos mueve el deseo de evitar que su pareja sufra daño si se infecta, por otro estamos obligados a hacer lo imposible por respetar el secreto profesional, por respeto a la legalidad vigente y por las consecuencias contraproducentes que tendría no hacerlo. Si hemos de romper el secreto, lo correcto será hacerlo en el mínimo grado que sea preciso. Debemos pues, utilizar toda nuestra capacidad de persuasión para evitar romper el secreto, si finalmente debemos hacer una excepción, que no norma, rompiendo el secreto en el grado mínimo, tendremos que hacer piruetas con el lenguaje para recordar a su pareja, "que toda relación sexual tiene riesgo, si sobre alguno de los miembros de la pareja existen dudas de sus hábitos", asegurándonos de que ha entendido plenamente su significado y ha comprendido como prevenir un potencial contagio. Esta situación de violación de la intimidad de una pareja y del secreto profesional, aun con riesgo legal, puede que sea éticamente necesaria, pero antes hemos de asegurarnos que esa situación de riesgo para la pareja es real, ya que si el paciente tomara todas las medidas para evitar la transmisión o no tuviera relaciones con ella, no se debería quebrar la confidencialidad.

La realización de la determinación de anticuerpos anti-VIH en todas las gestantes sin consentimiento previo, alegando la necesidad de prevenir la transmisión al feto, parece razonable, pero si analizamos sus consecuencias puede que dudemos de su bondad. $\mathrm{Si}$ a toda futura madre se le informa y pide consentimiento para realizarle la determinación y poder prevenir que su futuro hijo se infecte, es muy improbable que alguna se niegue, y es difícil justificar porque no informar a la gran mayoría basándonos en que puedan existir excepciones. Esos casos de "malas madres" podrían existir, si a ellas les realizamos sin su consentimiento el test y resultara positivo, será preciso comunicárselo y sabiendo nuestra forma de actuar desconfiaran. Siguiendo su lógica, por los mismos motivos que se negaron antes, es probable que tampoco deseen hacer el tratamiento o quieran someterse a una cesárea. Si no estamos dispuestos a llegar a un internamiento contra su voluntad o a forzar el tratamiento, y parece poco probable que consigamos una orden judicial para ello, ese supuesto beneficio 
para esos fetos brilla por su ausencia. Pero hemos generado tal clima de desconfianza en esas mujeres, que posiblemente nunca vuelvan por la consulta.

El argumento de que no es más necesario pedir un consentimiento informado para detectar seropositividad $\mathrm{VIH}$, que para hacerlo para el virus B de la hepatitis o la lúes, carece de fundamento. Ambas determinaciones no son comparables, ya que no tienen las mismas consecuencias socio-familiares, en el caso de ser positivo. El ser VIH-seropositivo margina y estigmatiza, cuando deje de tener ese matiz dejará de ser más necesario el consentimiento informado en esta enfermedad que en otras. Para los que no comprenden la diferencia quizá sea útil ponernos en su situación, de la cual nadie piense que está libre y menos el personal sanitario, que a nosotros o a nuestros familiares se les practique sin pedirles permiso, y sus resultados se acaben haciendo públicos en sus centros de trabajos, entre sus amigos o en el colegio de sus hijos.

Por estas razones expuestas, algunas de las consideraciones de Monés y Barrio (1) de tomarse literalmente como recomendaciones, bordean cuando menos la legalidad vigente, aunque nadie pueda dudar de su buena voluntad. Podemos estar muchos de acuerdo en que deberían modificarse ciertas leyes, y habrá que trabajar por conseguirlo, mientras los médicos no podemos incumplir sistemáticamente las leyes, elevar excepciones a la categoría de normas, caer en simplificaciones o confundir aspectos legales con aspectos éticos deseables. Los principios éticos legales son exigibles a todos y de obligado cumplimiento, los éticos solo se los puede exigir uno a si mismo, y si son contrarios a los legales no se puede pedir ni recomendar a los demás, aunque puedan ser muy deseables.

\section{J. C. Martín Escudero}

Servicio de Medicina Interna. Hospital Universitario del Río Hortega. Valladolid

1. Monés Xiol J., Barrio Medrano J.L. Aspectos éticos de la práctica médica en el SIDA. An Med Interna (Madrid) 2000; 17: 171-173.

2. Ley General de Sanidad (Ley 14/1986, de 25 de abril) Art. 10,6.

3. Constitución Española del 31 de Octubre de 1978. Art. 18.

4. Código Penal. Ley Orgánica 10/1995 de 23 de noviembre. Titulo III, Capitulo I: "Del descubrimiento y revelación de secretos" Art. 199 aptd. 1 y 2.

5. O. Parra García, M.B. Martínez Jarreta, M. Castellano Arroyo y R. Hinojal Fonseca. Sida. Problemas éticos y jurídicos de la relación médicopaciente a la luz del nuevo código penal. Med Clin (Barc.) 1998; 110: 186-190.

\section{Sarcoidosis nodular pulmonar}

\section{Sr. Director:}

Hemos leído con gran interés el artículo de Pérez de Llano y cols. sobre la sarcoidosis nodular pulmonar, publicado en su revista (1). Coincidimos con los autores en considerar esta forma radiológica de la enfermedad infrecuente, pero no excepcional. Aportamos un caso observado recientemente en nuestro centro.

Mujer de 32 años, sin antecedentes de interés, que consulta por un cuadro de dolor torácico izquierdo de características pleuríticas, de alrededor de mes y medio de evolución, de instauración brusca, que no había respondido a tratamiento con AINES instaurado por su médico de cabecera, sin otra sintomatología asociada. La exploración física era normal. La radiografía de tórax mostraba una atelectasia laminar en la base izquierda, y un ensanchamiento hiliar derecho sugestivo de adenopatías. El TAC torácico mostró adenomegalias localizadas fundamentalmente en hilio derecho, existiendo también adenopatías subcarinales, retrocava pretraqueales y prevasculares; asimismo se apreciaron tres nódulos pulmonares, el mayor de ellos de $1,5 \mathrm{~cm}$ de diámetro, periféricos, de bordes bien definidos, en lóbulo superior y medio derechos. El hemograma era normal y la VSG $=48$. La bioquímica sanguínea y el sedimento urinario eran normales, así como las pruebas de función respiratoria. Los anticuerpos antinucleares y anti-citoplasma de neutrófilo fueron negativos. El ECA se encontraba en valores normales, así como los marcadores tumorales (CEA, Alfa-FP y B-HCG). Se realizó broncoscopia con biopsia transbronquial sobre el LMD, intentándose asimismo obtener muestra de las adenopatías mediante punción transbronquial con aguja de Wang. La tinción de Ziehl fue negativa en todas las muestras de la broncoscopia, así como el cultivo de Löwenstein y la determinación de DNA genómico para M. tuberculosis. La biopsia mostró fragmentos de parénquima pulmonar normal, sin evidencia de granulomas. No se obtuvo material adecuado en la punción transbronquial. Se realizó biopsia de adenopatías mediastínicas mediante mediastinoscopia, demostrándose una linfadenitis granulomatosa no caseificante, morfológicamente compatible con sarcoidosis. No se observaron microorganismos con las técnicas de PAS y de Ziehl. La paciente quedó asintomática poco después de su ingreso, decidiéndose no iniciar tratamiento esteroideo. Un año más tarde la paciente sigue asintomática y un TAC de control mostró la resolución completa de las anomalías previas.

La sarcoidosis pulmonar es uno de los diagnósticos que deben considerarse en un paciente con aumento de tamaño de los ganglios linfáticos intratorácicos, con o sin anomalías asociadas en el parénquima pulmonar. Las adenomegalias generalmente afectan a las cadenas hiliar, traqueobronquial y paratraqueal, y por lo general son bilaterales y simétricas, contrastando con otras enfermedades como la tuberculosis o el linfoma de Hodgkin en que, cuando existe compromiso de los ganglios hiliares, éste suele ser unilateral (2). La aparición de nódulos pulmonares múltiples, de tamaño superior a $1 \mathrm{~cm}$ es infrecuente, especialmente en las series más antiguas, en las que se encontraba en alrededor del $2 \%$ de los casos (3). Probablemente el empleo generalizado de la TAC favorezca que esta forma de la enfermedad se reconozca más frecuentemente. Coincidimos con Pérez de Llano y cols. cuando consideran que este patrón radiológico en pacientes paucisintomáticos hace más factible el diagnóstico de sarcoidosis frente a otras enfermedades como tuberculosis, linfomas o metástasis, que suelen cursar con más síntomas, pero también estamos de acuerdo en que esta presentación radiológica atípica hace inexcusable obtener una muestra histológica para confirmar el diagnóstico. Nosotros hemos tenido la ocasión de observar un caso (ciertamente excepcional) de carcinoma pulmonar de células pequeñas presentado como nódulos pulmonares múltiples, en un paciente cuya única sintomatología era dolor torácico (4). Por otra parte, nuestra paciente sólo presentaba nódulos en un pulmón, cuando los nódulos pulmonares de la sarcoidosis suelen ser bilaterales (5-7).

Dado que, pese a todo, el primer diagnóstico de sospecha era sarcoidosis, se decidió realizar en primer lugar biopsia transbronquial, por tener ésta una alta rentabilidad en esta enfermedad, y ser relativamente incruenta. El resultado fue negativo, por lo que se realizó una biopsia ganglionar por mediastinoscopia, que dio el diagnóstico. Aunque podría sospecharse que los nódulos pulmonares tuvieran una etiología diferente a las adenopatías, la evolución radiológica paralela sugiere que formaban parte de la misma enfermedad. La biopsia transbronquial ha resultado ineficaz para el diagnóstico en algún otro caso de sarcoidosis pulmonar nodular bien documentada (8). 
En conclusión, estamos de acuerdo en que la sarcoidosis pulmonar nodular no es una forma radiológica excepcional, y que debe incluirse en el diagnóstico diferencial de los nódulos pulmonares múltiples, en particular si coexisten con adenopatías intratorácicas, y el paciente presenta escasos síntomas. La obtención de material para análisis histopatológico parece inexcusable. Al igual que en otros casos descritos, nuestra paciente evolucionó favorablemente sin tratamiento esteroideo, lo que indica que éste es un estadio precoz de la enfermedad, potencialmente reversible.

\section{R. Golpe Gómez, A. Mateos Colino, M. J. Armesto Fernández}

Unidad de Neumología y Servicio de Radiodiagnóstico. Hospital de Monforte de Lemos. Lugo

1. Pérez de Llano LA, Romeo de Araújo D, Soilán del Cerro JL, Ortiz Piquer M, González-Rosón Fernández O, Gago Hermida D. Sarcoidosis nodular pulmonar, ¿una forma radiológica de presentación infrecuente o una vasculitis granulomatosa? Descripción de tres casos. An Med Interna (Madrid) 2000; 17: 88-91

2. Fraser RG, Paré JAP, Paré PD, Fraser RS, Genereux GP. Enfermedad pulmonar de origen desconocido. En: Diagnóstico de las enfermedades del tórax $3^{\mathrm{a}}$ Ed. Fraser, Paré, Paré, Fraser, Genereux (Eds.) Editorial Panamericana. Buenos Aires 1992; 4: 2423-2521.

3. Kirks DR, Mc Cormick VD, Greenspan RH. Pulmonary sarcoidosis. Roentgenologic analysis of 150 patients. Am J Roentgenol Radium Ther Nucl Med 1973; 117: 777-86.

4. García Arangüena L, Sampedro Alvarez JR, Golpe Gómez R, García Pérez MM, Jiménez Gómez A, Mazorra Macho F. Carcinoma pulmonar de células pequeñas en forma de nódulos pulmonares múltiples. Arch Bronconeumol 1997; 33: 52-54.

5. Nakamura H, Kashiwabara K, Watanabe T, Yagyu H, Kiguchi T, Matsuoka K. Sarcoidosis with multiple nodular shadows in bilateral lung fields. Intern Med 1995; 34: 1144-5.

6. Kurogouchi F, Fujimori Y, Oharazawa A, Nagasaki M, Miyazawa K Sano K, Kubo K, Kiyosawa K. Sarcoidosis associated with multiple large pulmonary nodules. Respirology 1998; 3: 273-6.

7. Onal E, Lopata M, Lourenco RV. Nodular pulmonary sarcoidosis. Clinical roentgenographic and physiologic course in five patients. Chest 1977; 72: 296-300.

8. Paez Codeso F, Martín Villasclaras JJ, Miralles Lozano F. Nódulos pulmonares grandes como presentación de una sarcoidosis. Arch Bronconeumol 1998; $34: 227-8$.

\section{Marcadores tumorales en el cáncer de pulmón}

\section{Sr. Director:}

Hemos leído con interés el artículo de Haro y cols. (1) publicado, en su revista y quisiéramos hacer algunos comentarios.

En el artículo se pone de manifiesto el valor del antígeno carcinoembrionario (CEA), en suero, como marcador tumoral en el cáncer de pulmón. Pensamos que una de las muestras ideales para analizar estos marcadores en el carcinoma broncogénico es, pre- cisamente, el lavado broncoalveolar (LBA), pues consigue obtener material, directamente, de la zona donde asienta el tumor.

Estamos de acuerdo, al igual que Buccheri y cols. (2), con los autores (1), en que el uso de más de un marcador incrementa su rentabilidad diagnóstica. Nuestro grupo (3-7) ha evaluado en el LBA el comportamiento del CEA y de otras sustancias, antígeno polipeptídico tisular (TPA), antígeno polipeptídico específico tisular (TPS), enolasa neuronal específica (NSE) y los fragmentos solubles de la citoqueratina 19 (Cifra 21-1) (6). La elevación de las concentraciones de estos marcadores, con las limitaciones conocidas, implica una mayor probabilidad de que la lesión estudiada sea maligna. Esto, como es lógico, puede ser de ayuda en la orientación clínica del enfermo.

En cualquier caso, son pocos los trabajos sobre marcadores tumorales que tienen en cuenta la tipificación histológica y la estadificación del carcinoma pulmonar. Estimamos que esto es absolutamente fundamental para que estas sustancias puedan, definitivamente, tener su aplicación clínica. De igual forma, se ha demostrado la validez de los marcadores tumorales en la orientación pronostica, respuesta al tratamiento y capacidad para detectar recidivas del tumor (7).

Por todo lo expuesto consideramos que la investigación de los marcadores en el LBA tumorales ha de continuar. No obstante está aún por determinar el marcador o conjunto de marcadores los suficientemente específicos, pero también sensibles, que ayuden, de verdad, en la práctica clínica, en la valoración del cáncer de pulmón.

\section{F. J. Gómez de Terreros Caro, R. Alvarez-Sala Walther, C. Prados Sánchez, P. Díaz Ajero*}

Servicios de Neumología y *Cirugía Torácica. Hospital Univer sitario La Paz. Madrid

1. Haro M, Vizcaya M, Jiménez J, Quintanilla ML, Núñez A, Tomero A Utilidad del antígeno carcinoembrionario y otros marcadores tumorales en los pacientes con hemoptisis. An Med Intema (Madrid) 2000; 17: 186 191.

2. Buccheri GF, Ferrigno D, Sartoris AM, Violante B, Vola F, Curcio A. Tumor markers in broncogenic carcinoma. Superiority of tissue polypetide antigen to carcinoembryonic antigen and carbohidrate antigenic determinant 19-9. Cancer 1987; 60: 42-50.

3. Alvarez-Sala R, Blasco R, Callol L, Laguna R, Alvarez-Sala JL, Gómez de Terreros FJ. Tumour markers in broncho-alveolar lavage. Acta Oncol 1989; 28: 290-291.

4. Prados MC, Alvarez-Sala R, Blasco R, Chivato T, García Satué JL, García Río FJ, Gómez de Terreros FJ. The clinical value of neuron-specific enolase as a tumor marker in broncoalveolar lavage. Cancer 1994; 74: 1552-1555.

5. Prados C, Alvarez-Sala R. Valoración clínica de los marcadores tumorales en el lavado broncoalveolar. Med Clin (Barc) 1994; 102: 174-177.

6. Prados MC, Alvarez-Sala R, Terreros Caro FJ, Gómez L, Gómez de Terreros FJ, Villamor J. The concentrations of five tumour markers in both BAL fractions in lung cancer patients in relation to cigarrette smoking. Tumori 1999; 85: 454-457.

7. Prados C, Alvarez-Sala R, Gómez de Terreros J, Callol L, García Río F, Gómez Carrera L, et al. An evaluation of tissue polypeptide antigen (TPA) in the two bronchoalveolar lavage fractions of lung cancer patients. Jpn J Clin Oncol 2000; 30: 215-220. 